\title{
Numerical simulation with flow feature extraction of a propeller turbine unsteady rotor-stator interaction
}

\author{
J.-M. Gagnon \& C. Deschênes \\ Hydraulic Machinery Laboratory, Laval University, Canada
}

\begin{abstract}
We have seen many papers in the past few years reporting research on Francis hydraulic turbine flow. Interesting papers assessing the accuracy of the CFD numerical simulation were also presented for axial turbine draft tubes, as in the three Turbine 99 workshops. However, little knowledge is available on the flow taking place inside an axial turbine.

This paper focuses on the unsteady rotor-stator interaction in a propeller axial turbine. The flow behaviour is analysed at different rotor and stator relative locations with numerical simulations using a commercial code and $k$ - $\varepsilon$ turbulence model. The main goal is to study unsteady flow phenomena such as wake, separation, forces and pressure fluctuations in the propeller turbine. This investigation will help to design a series of flow measurements used in turn to improve future CFD simulations with realistic velocity profiles as boundary conditions.
\end{abstract}

Keywords: propeller turbine, numerical simulation, blade torque, partial load.

\section{Introduction}

Low head power plants are expected to be implemented increasingly in the future for economical, geographical and environmental purposes. Propeller turbines are well suited for these types of applications. They operate at higher flow rate, smaller head and faster rotational speed, thus being more compact than other types of machines. The US Department of Energy (DOE) is anticipating major growth for low head power plants [1] and studies such as Turbine 99 [2] or the work of Roussopoulos and Muntean [3, 4] show research trends on axial hydraulic machine. On a global perspective, optimization of size and weight 
towards highly compact system has led researchers to seek for a better understanding of fluid dynamic and unsteady effects. In turbomachinery applications, the interaction of stationary guide vanes and rotating runner may be considered as the source of unsteady phenomena.

Rotor-stator interactions taking place in turbines may be harmful for blades and surrounding systems as they induce pressure pulsations and torque variations on runner blades $[5,6]$. Fluctuations of the velocity field between the rotating and stationary parts also contribute to unsteadiness. Extensive literature is available on this subject for Francis turbines, pumps and pump-turbines. In [7], Page et al. analyse the time-averaged relative velocity profile and pressure fluctuations on a pump impeller outlet and a Francis turbine. Vu and Nennemann [8] have shown a method to analyse unsteady interactions in a Francis turbine using torque fluctuations and pressure spectrum. The unsteady torque on guide vanes pitching axis of a pump turbine in pump mode was predicted by Lipej et al. [9]. Recently, blade cracking and power plant failure were reported and due to the high dynamic stress of wicket gates and runner interaction [10]. In numerical simulation, Ruprecht et al. [11, 12] showed parallel computation capabilities to simulate a complete Francis Turbine including rotor-stator unsteady hydrodynamic effect. Performance of an in-house code to study the flow in an axial turbine with unequal pitch ratio was also addressed.

We can also gather great amounts of information on rotor-stator interaction and flow features inside axial turbines or compressors from the aerodynamic field. Basic phenomena such as separation, wake and secondary flows are similar when there is no shock and Mach number stay below 0.3. In this avenue, Zaccaria and Lakshminaryana [13] have exposed an extensive review of phenomena taking place in axial air turbine with an experimental investigation of the flow.

The present paper focuses on 3-D Navier-Stokes simulations of the flow in a propeller turbine using the commercial code ANSYS CFX. Three operating regimes near the best efficiency point are studied. Numerical methods are first detailed and then results from both steady and unsteady computations are analysed. Relevant quantities as torque on blades and pressure fluctuations are investigated, as well as wake characteristics behind guide vanes.

This research is carried out within the new Canadian Consortium in Axial Hydraulic Turbine taking place at Laval University, Quebec. Figure 1 illustrates a cut plane of the hydraulic propeller turbine along with future measurement access.

\section{Experimental overview}

Numerical simulations begin with the definition of different geometries of the domain and interfaces. This step allows us to look forward for the experimental setup that will be used to validate numerical results. Optical accesses for PIV and LDV measurements will be installed on critical regions, where the velocity and pressure fields need to be known to improve numerical simulations. CAD drawings are also used to help locating the calibration plate for PIV inside the distributor and for inter-blade PIV. 


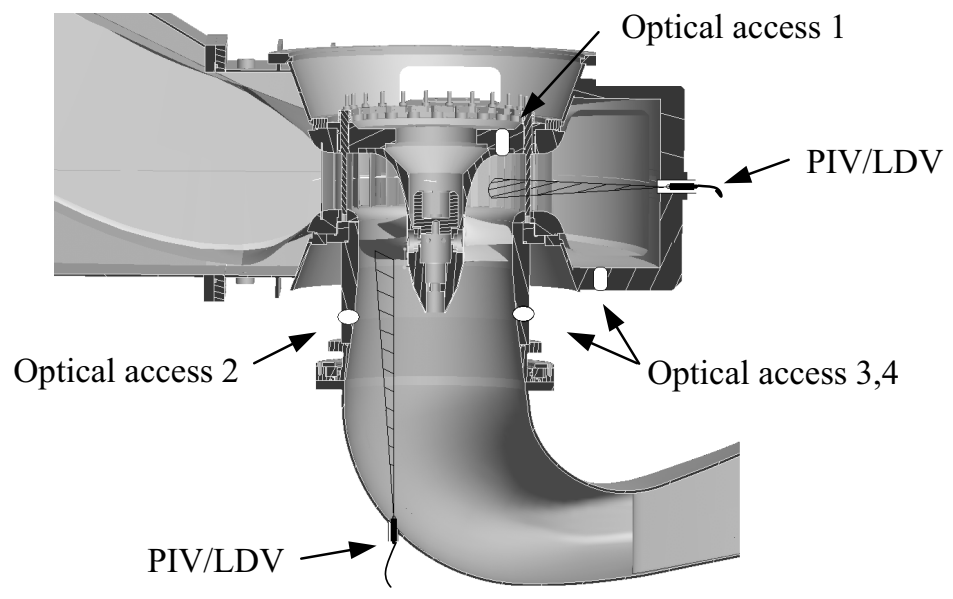

Figure 1: Cut plane of the hydraulic turbine with planned measurement concept.

\section{Computational methods}

We consider an incompressible unsteady 3-D turbulent flow in a single rotorstator passage of the model scale propeller turbine. RANS equations are used in combination with the $k-\varepsilon$ turbulence model and scalable wall functions accounting for unequal wall cells sizes and $\mathrm{y}^{+}$in the boundary layer calculation. The main advantage is to allow uses of coarser or finer mesh sizes and different turbulence models without having to remesh the whole boundary layer around runner blades and guide vanes. We use an advection scheme switching locally from a first order upwind differencing scheme to a second order scheme when necessary. Solver robustness is thus increased and convergence accelerated.

\subsection{Interface definition and analysis}

Several interfaces were used to connect stationary and moving meshes for steady and unsteady calculations. First, we estimated mesh performance and torque on the runner blade with a steady simulation averaging the circumferential velocity at the interface (Stage simulation). Since the effect of circumferential velocity is filtered through the averaging operation, it makes possible the use of a partial stator passage having only one guide vane and one stay vane instead of four. Total numbers of blades and guide vanes is respectively 6 and 24 for the whole machine, giving even pitch ratios. Other types of simulations were performed using a Frozen rotor for steady calculations and a Transient Rotor-Stator interface for unsteady calculations. Frozen rotor simulation cases were used as initial conditions for unsteady calculations. Figure 2 below shows interfaces definition: on the left with a stator pitch angle of 15 degrees in the azimutal plane for stage calculation and on the right with 60 degrees rotor-stator passage. 


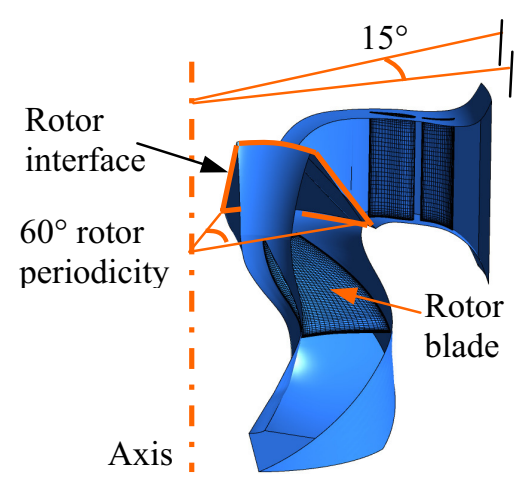

a)

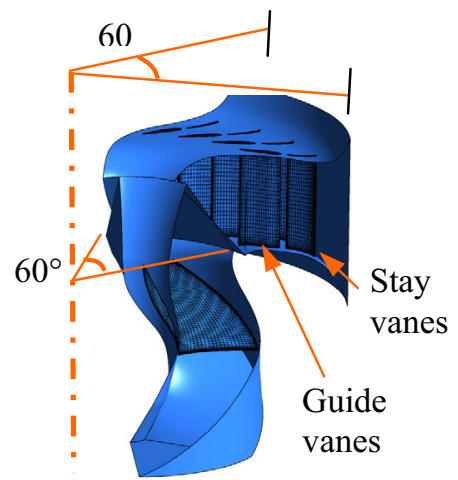

b)

Figure 2: $\quad$ Simulation domain and interface a) $15^{\circ}$ stator $-60^{\circ}$ rotor b) $60^{\circ}$ rotor/stator.
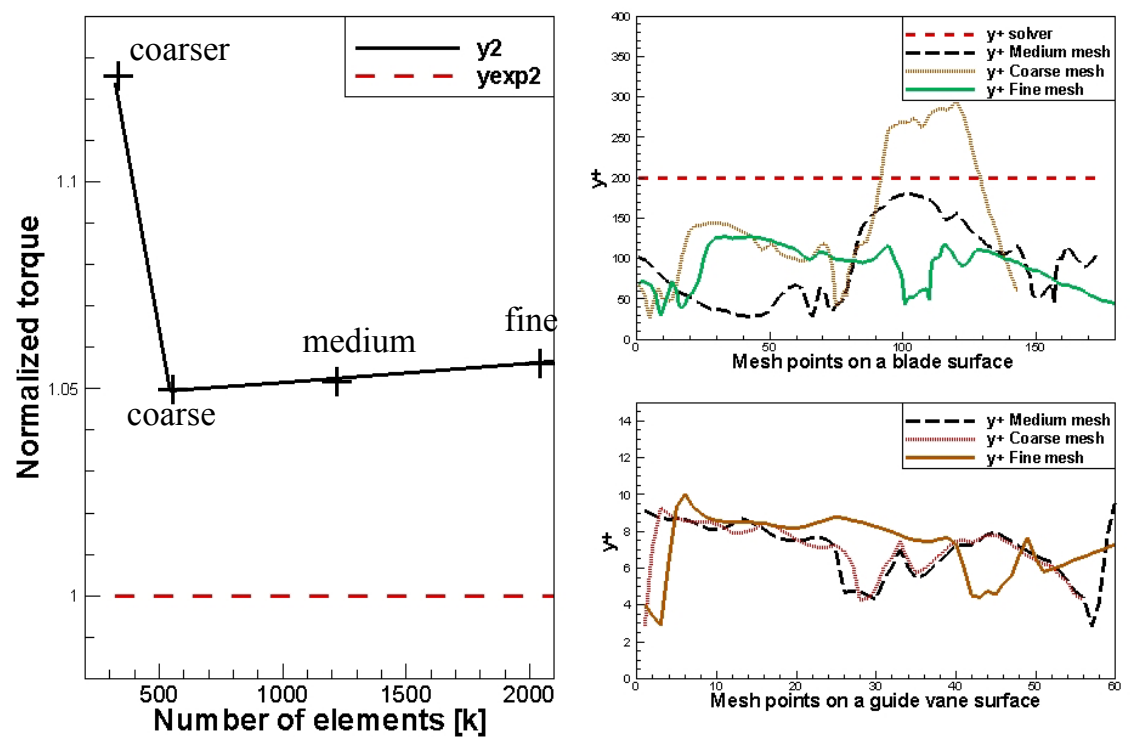

Figure 3: $\quad$ Torque in function of cells number (left), $y+$ distribution on blade and guide vanes walls (top and bottom right).

\subsection{Mesh independence}

We performed a mesh independence test to evaluate what size of mesh is needed for the type of flow phenomena which is analysed. In our case, the runner blade torque is the quantity investigated since it is directly linked to unsteady pressure fluctuations. Four mesh sizes were compared against computed torque values to ensure mesh independence. Figure 3 shows normalized runner blade torque in 
function of number of mesh elements (on left). Comparison of $\mathrm{y}+$ distribution for different mesh sizes was also done on guide vanes and runner blade walls. The lowest blade normalized torque value of 1.05 corresponding to the coarse mesh computation gives the best computed torque compared to experimental data. However, having a look at the right figures, this mesh exceeds the recommended maximum $y+$ value of 200 on the runner blade.

This may lead to poor boundary layer calculations. Therefore the medium grid of $1000 \mathrm{k}$ elements has been chosen for all calculations at peak, partial and overload conditions to fulfil the solver boundary requirement. Note that no calculations got closer to $5 \%$ of the experimental torque value, leaving place for numerical simulation improvement.

\section{Rotor-Stator interaction analysis}

Unsteady rotor-stator interactions may be attributed to instantaneous pressure fluctuations. Wakes building along the boundary layer of stay vanes and guide vanes of the distributor are other unsteady phenomena which may be considered. These flow phenomena are weaker in intensity and reflect higher harmonics on the frequency spectrum. Results from steady state simulation using a Frozen rotor interface are presented in this section for wake interaction. Dissipation of the wake is investigated with the following indicators: the turbulent kinetic energy, the vorticity and the velocity profile. Figure 4 shows contours of turbulent kinetic energy and vorticity in a blade-to-blade view for three operating regimes. Here, $\alpha^{*}$ is the normalized opening guide vane angle equal to the ratio of the actual opening angle to the angle corresponding to the best efficiency point, $\alpha / \alpha_{\text {peak }}$. Note that the flow velocity, $U_{\infty}$, changes direction across interfaces accounting for rotation terms in N.-S. equations.

Figure 4 presents two periodic profiles having a total of eight guide vanes and two runner blades. The blade-to-blade planes of cases shown in Figure 4a) are located at low span, near the shroud, to capture the small gap between guide vanes and runner blades. From the contours, we can distinguish a slight increase in $k$ across the interface with increasing guide vane opening (from left to right, light grey contour). At overload condition ( $\alpha^{*}=1.15$, top right), the contour of $k$ almost reaches the blades and may indicate the possibility of wake interaction with runner blades. The level of wake kinetic energy is low for all cases and around $0.03 \mathrm{~m}^{2} / \mathrm{s}^{2}$.

In figure $4 \mathrm{~b}$ ), the slice is at mid span and one can see that wakes behind guide vanes and runner blades induces regions of high radial vorticity (dark contour). The vorticity intensity is kept high across the interface and propagates until it reaches runner blades surfaces for all cases. A further investigation of these steady state calculations gives insight about how far gradients of velocity are convected into the rotor domain.

In Figure 5, one can see a typical case of how velocity profiles on circular lines in the rotor-stator passage are gradually dissipated. Velocity gradients are dissipated up to $99 \%$ before reaching the blade at peak condition. 


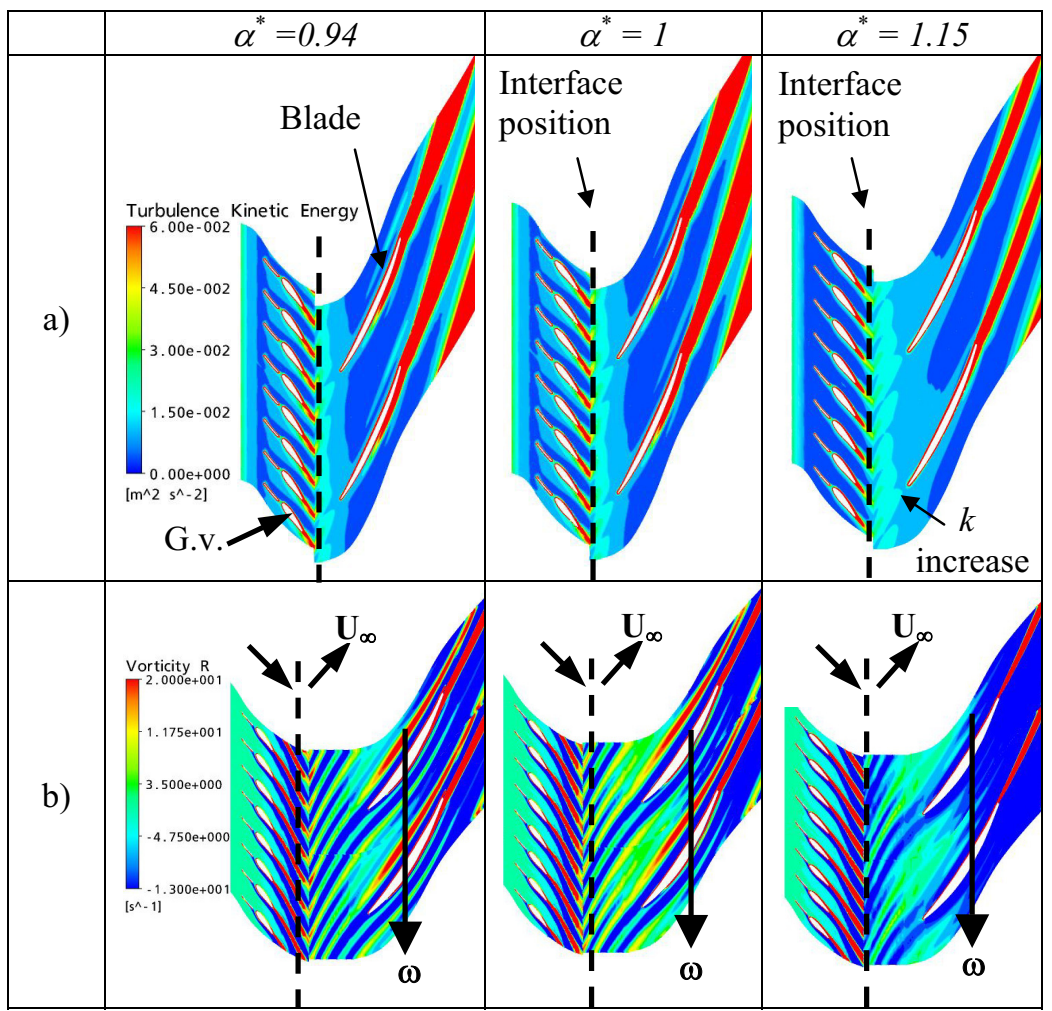

Figure 4: Blade-to-blade contours of a) turbulent kinetic energy b) radial vorticity.
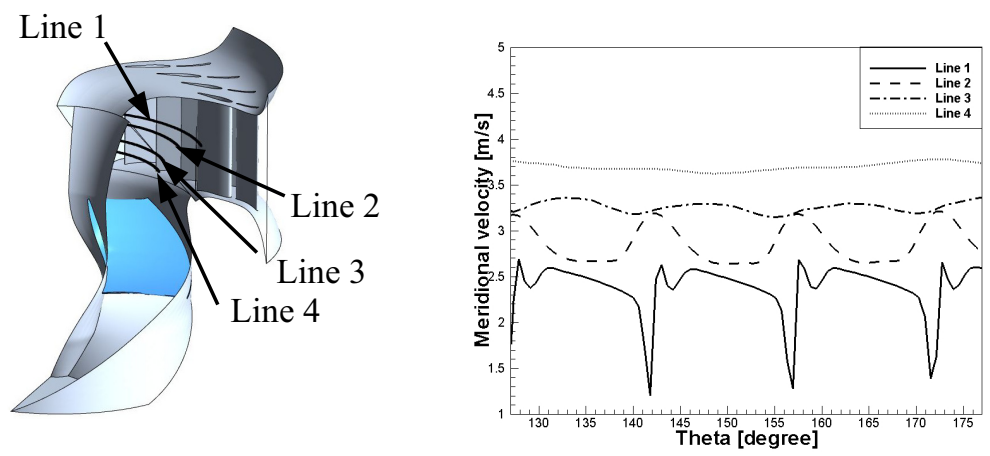

Figure 5: Meridional velocity profiles along different streamwise stations in the rotor-stator passage. 


\section{Unsteady rotor-stator analysis}

Unsteady interactions of runner blades with stationary guide vanes have been studied by looking at the frequency spectrum of instantaneous force, torque and pressure signals over time at various locations. Figure 6 illustrates the time and frequency signal of drag force on each of the four guide vanes in the distributor.

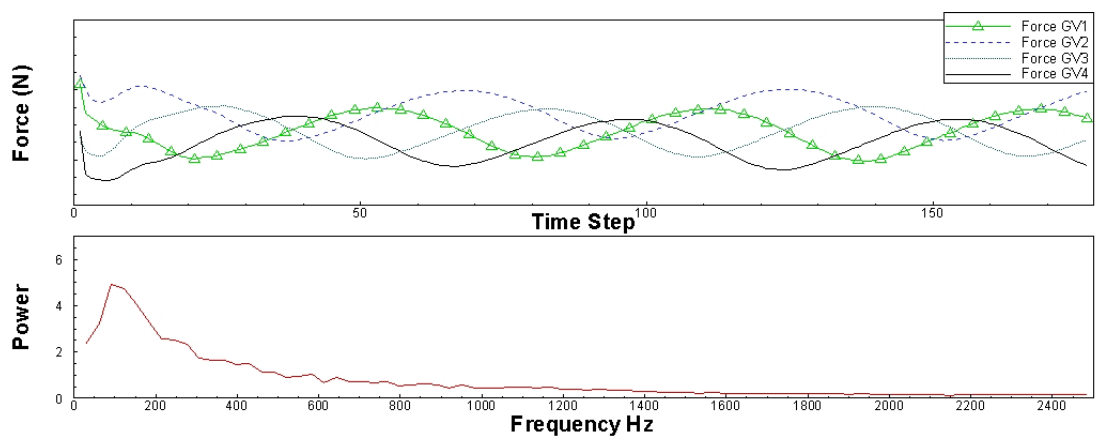

Figure 6: $\quad$ Guide vanes force for $\alpha^{*}=1$ with signal in time (top) and frequency spectra (bottom).

Fluctuations of instantaneous forces on the guide vanes are about $5 \%$ of the average force. The peak on the frequency spectrum corresponds to the blade passing frequency, $f_{b}=N_{b} \cdot f_{\omega}$, where $f_{\omega}$ is the rotor frequency and $N_{b}$ the number of blades. The phase shift between two adjacent force signals equals $2 \pi / N_{g v}$, where $N_{g v}$ is the number of guide vanes.

We investigated the instantaneous blade torque on the rotor side to assess the effect of transient interactions on the runner in the rotating frame of reference. In figure 7, the three operating regimes are shown with a FFT transform applied on the same amount of cycles for each torque signal.

Amplitude peaks for all regimes correspond to the wicket gate passing frequency, $f_{g v}=N_{g v} \cdot f_{\omega}$. Wake interactions have very small effects on the unsteady torque since there is no other peak at higher frequencies. Also, we see that the amplitude of the signal at overload condition $\left(\alpha^{*}=1.15\right)$ is higher than in the other cases confirming the fact that pressure fluctuations effects on blades and on other unsteady phenomena are increased as we move away from peak condition. Fluctuations of the torque are small and estimated about $0.3 \%$ of the time-averaged torque.

To complete this analysis, we have evaluated the pressure signals from two points located very close to the interface at mid span. Figure 8 shows the time and frequency signals for these two points. Similar conclusions applied as for the torque and forces analysis and we see that pressure frequencies match with $f_{b}$ for the stator point and $f_{g v}$ for the rotor point. One can also find additional higher harmonics around 7 and 14 times $f_{b}$ (top axis, bottom left figure). These 
harmonics are likely due to numerical noise rather than to unsteady interactions (wakes, von Karman vortices). Further investigations are needed to better understand the phenomenon.

Pressure fluctuations around the time-averaged signals are about $2.4 \%$ and $0.5 \%$ respectively for the stator and the rotor points.

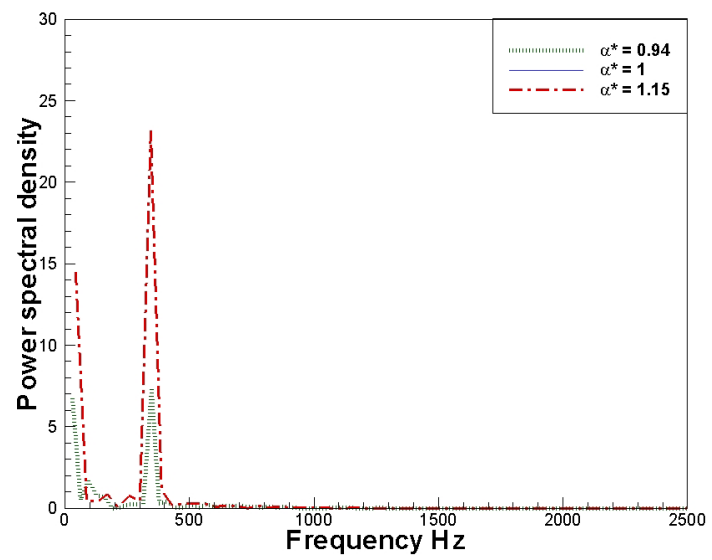

Figure 7: Frequency spectra of torque on runner blades.
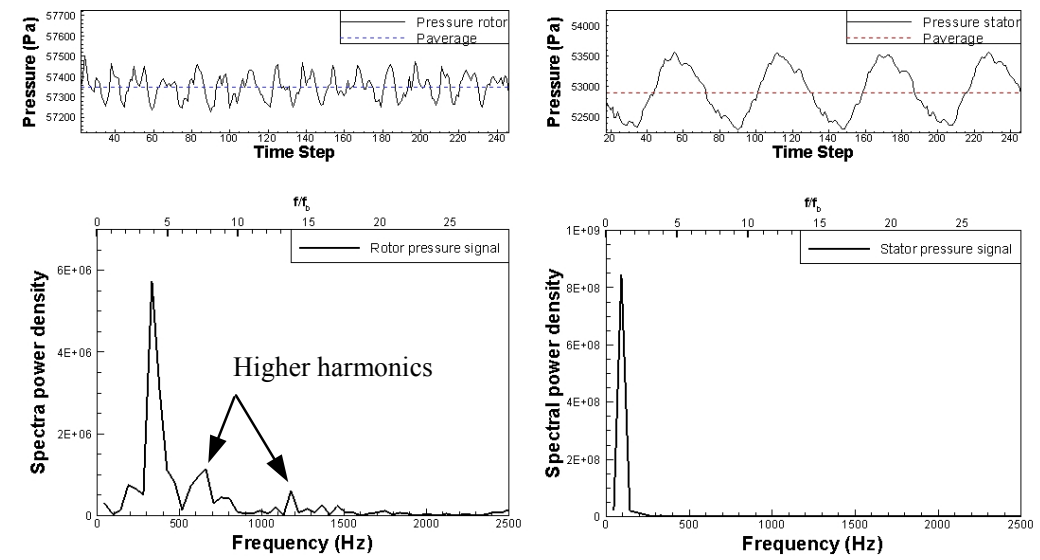

Figure 8: Pressure time and frequency signal of two points situated close to the interface.

\section{Conclusion}

In this paper, we studied wakes and unsteady rotor-stator interactions for different operating regimes of a propeller turbine. The wake behind guide vanes is dissipating very fast. It is still unsure whether it has an effect on the rotor- 
stator unsteady interactions since higher harmonics of the spectrum were not well defined. The main interaction is therefore attributed to the pressure field fluctuations. Peak amplitudes correlate with the runner frequency for all pressure related signals. Although it was found that unsteady forces, torque and pressure fluctuations were weak in amplitude compared to time-averaged values (below 5\%), one must keep in mind that current computations were done at model scale. Same types of study on Francis turbine indicate that fluctuations might double at prototype scale. Finally, we are really looking forward the upcoming experimental phase to increase our knowledge and understanding of the propeller turbine flow.

\section{Acknowledgements}

The authors would like to thank Hydro-Quebec and Alstom Hydro Canada Inc. for their financial and technical supports. Their contribution for turbine geometries and experimental model is of great importance to this research project. Richard Fraser, Maryse Page, Sebastien Houde and Michel Sabourin are also gratefully acknowledged for their time and devotion to the project.

\section{References}

[1] Douglas, G. H., Kelly, S.R. (2006), Feasibility Assessment of the Water Energy Resources of the United States for New Low Power and Small Hydro Classes of Hydroelectric Plants, U.S. Department of Energy, DOEID-11263.

[2] Andersson U., Karlsson R. (1999), Quality aspects of the Turbine 99 draft tube experiments, Proceeding of Turbine 99 - workshop on draft tube flow, Technical report, Lulea University of Technology, Sweden.

[3] Roussopoulos K., Monkewitz P.A. (2000), Measurements of Tip Vortex Characteristics and the Effect of an Anti-Cavitation Lip on a Model Kaplan Turbine Blade, Flow, Turbulence and Combustion, Netherlands, vol. 64, p. 119-144.

[4] Muntean, S., Balint, D. (2006), Analytical representation of the swirling flow upstream the Kaplan turbine for variable guide vane opening, XXIII IAHR Symposium, Yokohama.

[5] Nennemann, B., Vu, T.C., Farhat, M. (2005), CFD prediction of unsteady wicket gate-runner interaction in Francis turbines: A new standard hydraulic procedure, Hydro 2005.

[6] Ciocan, G.D., Kueny, J.L. (2006), Experimental Analysis of Rotor-Stator Interaction in a Pump-Turbine, XXIII IAHR Symposium, Yokohama.

[7] Page, M. Théroux, E., Trépanier, J.-Y. (2004), Unsteady rotor-stator analysis of a Francis turbine, XXII IAHR Symposium on Hydraulic Machinery and System, Stockholm, Sweden.

[8] Vu, T.C., Nennemann, B. (2006), Modern trend of CFD application for hydraulic design procedure, XXIII IAHR Symposium, Yokohama. 
[9] Lipej, A., Jost, D., Meznar, P. (2006), Numerical analysis of rotor-stator interaction in a reversible pump-turbine - pump mode, XXIII IAHR Symposium, Yokohama.

[10] Coutu, A., Proulx, D., Coulson, S., Demers, A. (2004), Dynamic Assessment of Hydraulic Turbines, Proceedings of HydroVision 2004, Montreal, Quebec, Canada, August 16-20.

[11] Ruprecht, A., Heitele, M., Helmrich, T. (2000), Numerical Simulation of a Complete Francis Turbine including unsteady rotor/stator interactions, XX IAHR Symposium, Charlotte, North Carolina.

[12] Ruprecht, A., Bauer, C., Gentner, C., Lein, G. (1999), Parallel Computation of Stator-Rotor Interaction in an Axial Turbine, ASME PVP Conference, CFD Symposium, Boston.

[13] Zaccaria, M. and Lakshminarayana B. (1997), An experimental investigation of steady and unsteady flow field in an axial flow turbine, NASA contractor report; 4778, National Aeronautics and Space Administration. 